\title{
Accuracy and precision of matching inspired lung volume
}

\author{
JEFFREY A. GLINER, LAWRENCE J. FOLINSBEE, and STEVEN M. HORVATH \\ Institute of Environmental Stress, University of California, Santa Barbara, California 93106
}

\begin{abstract}
Three questions were asked: How accurately can lung volumes be reproduced? Do the underlying sensory continua of inspired lung volume subscribe to Weber's law? Are planned inspirations more accurately reproduced than constrained inspirations? The ability to match inspired lung volume was investigated utilizing the psychophysical method of average error. Fourteen subjects participated in two different conditions in order to answer these questions. The results indicated that inspired lung volumes were reproduced much more accurately than had been previously reported. The sensitivity of change for planned inspirations was identical to that of constrained inspirations $(k=.018)$. However, the absolute threshold was significantly greater for the constrained group. The underlying sensory continua subscribed to a generalized version of Weber's law. These results are discussed within the context of our present knowledge of receptor information of movement.
\end{abstract}

The respiratory system in humans operates under both conscious and unconscious control. Most respiratory alterations are regulated automatically, usually without awareness of the changes. However, under certain circumstances, such as anxiety-inducing situations, increases in respiration may be noticed. A change in ventilation is normally accomplished by altering both the volume of inspiration per breath (tidal volume) and the frequency of breathing. In order to alter the inspired volume, the ability to detect a difference between the previous volume and the volume to be inspired must be present. In addition to this detection process, the organism must be able to control the system to produce a new inspiration suitable to that specified by the detection system. These two processes, detection and control, may not be independent. The purpose of this research was to investigate the ability to detect and control inspiratory volume in humans.

The role of sensitivity or accuracy of detection of lung volume has been investigated. West, Ellis, and Campbell (1975) manipulated changes in tidal volume by adding carbon dioxide, lowering the fraction of inspired oxygen, or encouraging changes in inspiration relative to a visual target stimulus. When tidal volume increased by approximately $700 \mathrm{ml}$, the subject perceived an increase in ventilation. This just perceptible change in tidal volume was similar for both large and small volumes, indicating that the just noticeable differences (JNDs) were constant over a

This work was supported in part by Environmental Protection Agency Grant EPA R804853 and National Institutes of Health Grant NIH HL 26034. Correspondence and reprint requests should be addressed to Jeffrey A. Gliner, Institute of Environmental Stress, University of California, Santa Barbara, California 93106. range of tidal volumes. The average perceived change of $700 \mathrm{ml}$ found in that study seemed relatively insensitive, considering the complex integration between the motor cortex and the pulmonary system and also the complex motor control involved in the interchange between speech and breathing. Furthermore, Bakers and Tenney (1970) found that the exponent of the power function relating perception of inspired lung volume to the actual inspired volume during magnitude estimation and production experiments averaged 1.3. This would imply a rather sensitive sensory system (Baird \& Noma, 1978).

The ability to reproduce an inspiration may involve control processes different from the ability to perceive a difference between two inspirations. This question was partially addressed by Bakers and Tenney (1970). They used both magnitude estimation and magnitude production techniques and found that the exponents for volume estimation were not different from volume production for either inspiration or expiration. This was somewhat unexpected, since presumably different control processes underlie each type of activity. For example, magnitude production allows the subject to preplan the termination of the movement, whereas the experimenter determines the termination of the movement in magnitude estimation experiments. Studies on movement extent, which may involve some of the same underlying sensory information as inspired lung volume, have found that reproduction of arm movements was more accurate when the subject selected his own termination point as compared with when this point was determined (constrained) by the experimenter (Stelmach, Kelso, \& McCullagh, 1976). This would imply different sensitivities or Weber fractions as a function of the locus of control of the response. Preplanning of inspira- 
tion may account for differences in the ability to finely control respiratory movements. On the other hand, it is possible that the sensitivity may not be different between these two different control processes, as indicated by Bakers and Tenney (1970), but that there may be differences in the absolute threshold.

Three different questions were addressed in this study: How sensitive is the detection of inspired lung volume relative to other sensory systems? Are planned inspirations more accurately reproduced than experimenter-constrained inspirations? Do the underlying sensory continua of inspired lung volume subscribe to Weber's law?

\section{METHODS}

\section{Subjects}

Fourteen volunteers (seven males and seven females) between the ages of 21 and 35 participated in this study. The procedures were described to each subject, and each gave his/her informed consent to participate. ${ }^{\prime}$ All were employed at the Institute of Environmental Stress and had had some previous experience with pulmonary function apparatus. Each subject performed the task 10 different times, each time on a separate day.

\section{Procedure}

The subject was seated in the laboratory, with opaque goggles over his/her eyes to eliminate any visual cues the volume bags or spirometer might provide to enhance the perceptual process. Three different volumes were selected that represented $25 \%, 50 \%$, and $75 \%$ of the subjects' inspiratory capacity while sitting. The psychophysical technique-the method of average error (Woodworth \& Schlosberg, 1965)-was employed in these studies. For each trial in Condition 1 (constrained inspiration), the subject breathed normally and, when ready, turned a valve and inhaled the standard stimulus from a breathing bag. At the end of this inspiration, the subject exhaled to approximately the same point (FRC-functional residual capacity) as prior to the inspiration, returned the valve to its original position, and then inspired a volume that was as close as possible to the standard stimulus. This volume was recorded on the spirometer and was later compared with the known volume of the bag. Each subject performed 30 trials per day (10 of each volume, presented randomly) for 5 days. Prior to the first day, the subject practiced until completely familiar with the instructions and apparatus. For Condition 2 (planned inspiration), the same three inspired volumes were used for each subject as in Condition 1. However, in this condition, the subject did not inspire a standard volume from a bag, but produced his own standard and then tried to match it. Prior to each trial, the investigator instructed the subject as to which volume-small, medium, or large-he wished the subject to inspire. On the next breath, the subject was requested to reproduce this standard as closely as possible. In order for the subject to reproduce standards that corresponded approximately to $25 \%, 50 \%$, and $75 \%$ of their inspiratory capacity, the investigator occasionally prompted the subject, suggesting either increases or decreases in the size of the standard. (Note that these increases or decreases informed the subject only of the size of his standards and had no information value as to the accuracy of the comparison stimulus. Thus, no knowledge of results was provided.) In the interval between the standard breath and the comparison breath, the subject turned a valve. The reason for this was to standardize the procedure between Conditions 1 and 2 , because turning of the valve might set up a condition of divided attention for the subject. Each subject performed 30 trials per day ( 10 of each volume, presented randomly) for 5 days. Half the subjects started with Condition 1 and then performed Condi- tion 2, while the other half started with Condition 2 and then performed Condition 1.

\section{Apparatus}

Inspired volume was recorded on a water-filled spirometer (Collins respirometer). For Condition 1 of the study (constrained inspiration), inspired volumes were breathed from gas bags attached to a large-bore three-way valve. A calibrated 3-liter syringe was used to deliver a known volume to an evacuated 5 -liter gas bag. The same three-way valve was used for the second part of the study, but the subject inspired directly from the spirometer.

\section{RESULTS}

Table 1 describes the mean volumes of the standard stimuli for the constrained and planned conditions for $25 \%, 50 \%$, and $75 \%$ volumes. Also, the maximum volumes, obtained during the screening pulmonary function test, are presented. The reason for this increase in size of the standard for the planned condition was presumably the inability of the investigator to shape the standard breaths to the size identical to that used in the constrained condition.

Three different measures were derived from the spirometer tracings. Absolute error or average error was calculated as the sum of the error scores (error was the difference between the standard and comparison stimuli) divided by the number of trials, with the sign being disregarded. Constant error or algebraic error was determined in a similar manner, except the sign of the error was included. The difference limen, or JND, was calculated as $.6745 \times \mathrm{SD}^{2}$, where SD is the standard deviation of a number of error scores for a subject. All analyses were performed by either a three-factor repeated measures analysis of variance (type of movement by size of movement by days) or a two-factor repeated measures analysis of variance (type of movement by size of movement). When significant interaction effects were observed, simple main effects analyses were performed. NewmanKeuls post hoc tests were then performed on all significant main effects and simple main effects.

In order to determine whether there were any systematic differences over time on any of the measures (e.g., familiarity with the equipment or practice), a three-factor analysis of variance, as specified above, was carried out on the three dependent measures.

\section{Table 1}

Group Mean and Standard Deviation (in Milliliters) of the Standard Breaths for $25 \%, 50 \%, 75 \%$, and Total Inspiratory Capacity (IC)

\begin{tabular}{rrrrrr} 
& \multicolumn{2}{c}{ Constrained } & & \multicolumn{2}{c}{ Planned } \\
\cline { 2 - 3 } \cline { 5 - 6 } IC & Mean & SD & & Mean & SD \\
\hline $25 \%$ & 743 & 155 & 979 & 328 \\
$50 \%$ & 1478 & 299 & & 1539 & 352 \\
$75 \%$ & 2193 & 474 & & 2340 & 455 \\
$100 \%$ & 2971 & 622 & & 2971 & 622 \\
\hline
\end{tabular}


The results showed a significant Days by Type of Inspiration interaction $[F(4,52)=4.74, p<.01]$ for the constant error. This was due to an increase in constant error over the last 3 days for the constrained movement group. There was also a significant Days by Type of Inspiration by Volume of Inspiration interaction $[F(8,104)=3.16, p<.01]$ for absolute error. The absolute error for the unplanned inspiration group increased over days for small inspirations and decreased over days for large inspirations. No other effects due to days (time) were observed. For subsequent analyses, all measures were summed over 5 days, giving each size of inspiration a total of 50 trials within each condition per subject. Two-factor analyses of variance (type of movement by volume of inspiration) were then carried out.

Figure 1 presents the results of the absolute error for the planned and constrained movements at each of the inspirations. A significant main effect was observed for type of inspiration $[F(1,13)=27.34, p<.01]$, indicating that the absolute error was larger for the constrained than for the planned movements. There was also a significant Type of Inspiration by Volume of Inspiration interaction $[\mathrm{F}(2,26)=4.11, \mathrm{p}<.05]$, indicating that for the constrained movements, absolute error was significantly larger for small inspirations than for medium inspirations.

The constant error allows interpretation of the direction of the error of the inspiration. Figure 2 shows the average constant error as a function of type of

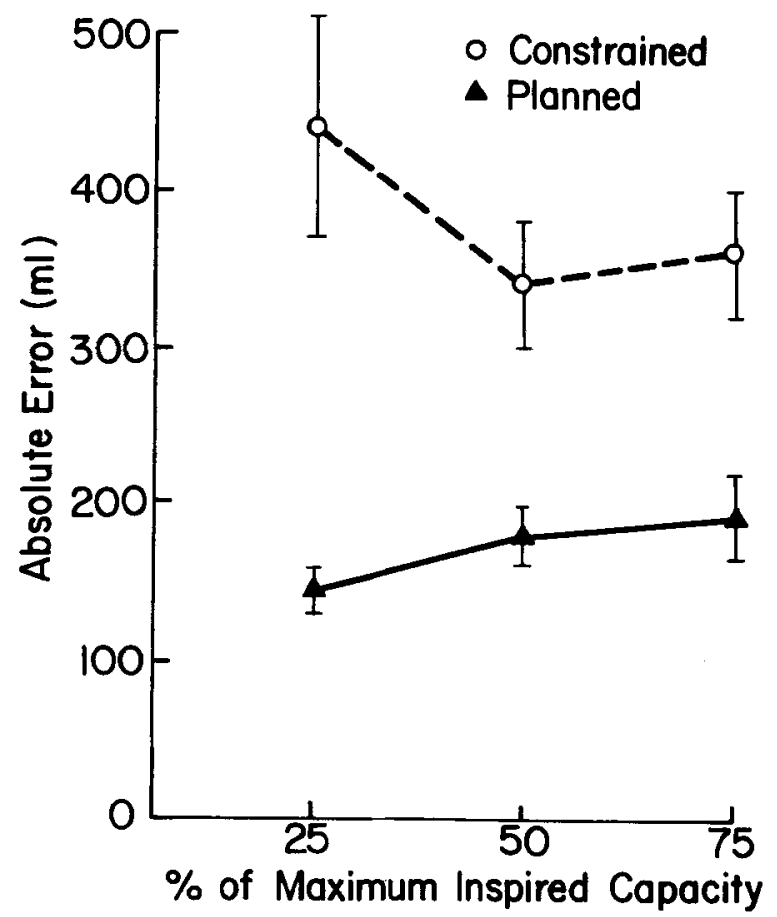

Figure 1. Mean absolute error for planned and constrained inspirations for $25 \%, 50 \%$, and $75 \%$ of maximum inspiration.

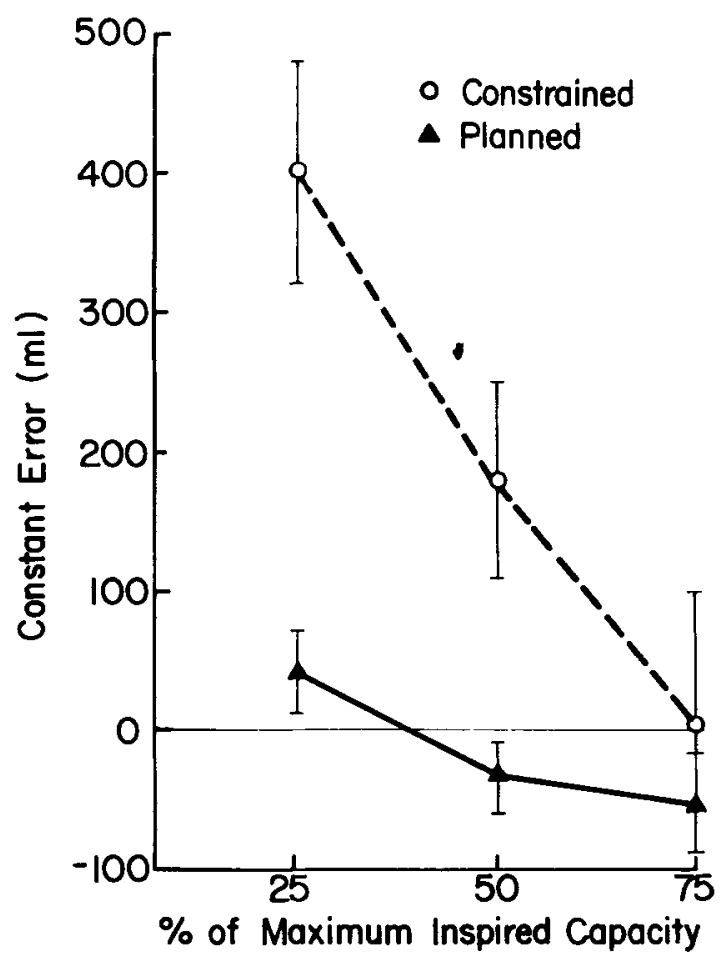

Figure 2. Mean constant errors for planned and constrained inspirations for $\mathbf{2 5 \%}, \mathbf{5 0 \%}$, and $75 \%$ of maximum inspiration.

breath and size of breath. A significant main effect for the type of inspiration $[F(1,13)=8.117, p<.05]$ indicated that constant error was larger and positive for the constrained inspirations, whereas the constant error for the planned inspirations was negative. Thus, subjects had a tendency to overestimate the standard for constrained inspirations and underestimate the standard for planned inspirations. A significant Type of Inspiration by Volume of Inspiration interaction $[F(2,26)=14.51, p<.05]$ indicated that, for constrained inspirations, the constant error significantly decreased as the size of the inspirations became larger. On the contrary, for planned inspirations, the constant error was less for small inspirations than for medium and large inspirations.

The just noticeable difference describes the amount of change of inspiration needed to perceive a difference. Figure 3 shows the JNDs for planned vs. constrained inspirations at the three different volumes. A significant main effect was observed for the type of inspiration $[F(1,13)=68.18, p<.01]$, indicating larger JNDs for the constrained condition. A significant main effect was also observed for the size of inspiration $[F(1,13)=5.59, \mathrm{p}<.05]$. Small inspirations were more accurately reproduced than were medium and large inspirations.

In order to determine whether the underlying sensory continua subscribed to Weber's law, the data points for the planned and constrained conditions (Figure 3 ) were fitted by a generalized version of 


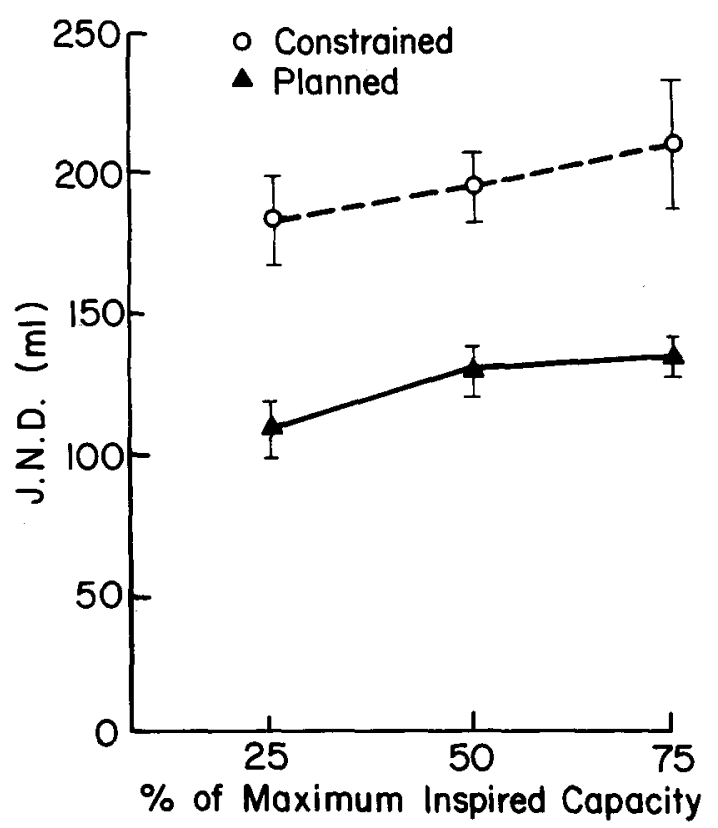

Figure 3. Mean JNDs for planned and constrained inspirations for $25 \%, 50 \%$, and $75 \%$ of maximum inspiration.

Weber's law, $\Delta \mathrm{I}=\mathrm{KI}+\mathrm{C}$ (Baird \& Noma, 1978), where $\Delta \mathrm{I}$ was the JND, I was the stimulus intensity, $\mathrm{C}$ was proportional to the absolute threshold, and $\mathrm{K}$ was the Weber fraction. The functions computed from the mean JNDs were as follows: $\Delta I=.018 \mathrm{I}+95$ (planned); $\Delta \mathrm{I}=.018 \mathrm{I}+170$ (constrained). The Weber fractions for the two conditions were identical, and the data fit the generalized form of Weber's law. However, the $y$ intercept or absolute threshold was almost twice as large for the constrained condition as for the planned condition.

\section{DISCUSSION}

The ability to match lung inspiration changes was more than twice as sensitive as that previously reported in the literature (West et al., 1975). The difference was most likely accounted for by the different procedure utilized in the present experiment. West et al. (1975) utilized a considerably longer time interval between the standard and comparison stimuli, and consequently memory recall may have played a distinct role in the discrimination between the two stimuli, causing a less sensitive discrimination. Furthermore, the comparison stimuli were gradually changing in that study, causing a temporal adaptation. The Weber fraction obtained for our study for either planned or experimenter-constrained lung volumes was .018 , which was quite sensitive as compared with other common continua (Baird \& Noma, 1978). Although this was the only study that investigated JNDs for inspired lung volume, Bakers and Tenney (1970) utilized magnitude estimation and production with the same sensory system. Their finding of an average exponent of 1.3 indicated a relatively sensitive system. We computed what the exponent would be from our data by the formula $n=h / k$, where $\mathrm{n}$ was the exponent and $\mathrm{h}$ and $\mathrm{k}$ were the Weber fractions for numbers and the stimulus continuum, respectively (Baird \& Noma, 1978). Utilizing $h$ as equal to .03, as developed empirically by Teghtsoonian (1971), we arrived at an exponent of 1.67 , which was greater than that found by Bakers and Tenney (1970) but identical to data from our laboratory for magnitude estimation and production (Mihevic, Gliner, \& Horvath, Note 1). Also, the high sensitivity of this fraction would predict a short dynamic range for this sensory continuum (Teghtsoonian, 1971). The dynamic range for this function is slightly over one order of magnitude from absolute threshold to maximum lung inspiration, making this system relatively short as compared with other systems that have previously been investigated, thus confirming Teghtsoonian's prediction.

The Weber fractions for the two different experimental conditions-constrained and planned-were identical. This added support to the Bakers and Tenney (1970) finding that magnitude estimation and production experiments did not produce different exponents, even though estimation experiments could be considered constrained by the investigator. Thus, preplanning of the movement changed the absolute threshold in favor of a smaller threshold, but once above threshold, the detection of differences was of equal sensitivity. A close examination of the constrained condition added further explanation for this finding. The subject received the three different volume bags in random order; thus, for any given standard, the subject always had to take a large inspiration on the possibility that the standard was $75 \%$ of maximum inspired capacity. However, in one-third of the cases, the standard was only $25 \%$ of maximum. This sudden termination of the inspiration would make it especially difficult to reproduce this volume size. This was demonstrated by larger absolute errors for the constrained condition than for the planned condition, and also, within the constrained condition, the $25 \%$ volume showed larger absolute errors than either of the other two volumes. Not only were the errors larger for the constrained condition, but they were also overestimated, as would be expected by our explanation of sudden termination of the breath. This overestimation decreased as the size of the volume increased. The constant error for the planned condition reversed sign as the volume increased, although the absolute size of the error was the same. Therefore, the unpredictability of the constrained situation caused the subject to always take a deep breath prior to inhaling the standard, introducing noise into the system and resulting in a greater absolute threshold for this condition. This noise would 
add a constant to the steadily increasing noise inherent in the system as the size of the inspired volume increased, as suggested by Weber's law.

The finding that our data subscribed to Weber's law when the absolute threshold was taken into account might provide some clues as to the mechanisms underlying this system, if one assumes that subjective responses are interpreted as a function of peripheral receptor processes. Thus, a constant Weber fraction might imply an increase in receptor firing as the size of the movement or volume increased. The role of joint receptors in sensing movement position, suggested earlier, apparently must be revised (Matthews, 1977; McCloskey, 1978). This decision becomes evident for midrange movements in which these receptors do not provide sufficient detailed information to account for observed proprioceptor acuity (McCloskey, 1978). Instead, primary and secondary endings of the muscle spindles have been postulated as providing the information needed to subserve the sense of position or movement. Specific to inspiratory volume, these receptors would lie in the intercostal muscles and diaphragm. However, it is also possible that other sources of receptor information could provide cues to accurately reproduce lung volume. Among these, tactile receptors from the skin for large breaths might play a major role, since most subjects indicated trying to reproduce a feeling of position under this condition. Other possibilities include sensation of abdomen position or stimulation of costovertebral joint receptors (Axen \& Haas, 1979; Shannon, 1979). On the other hand, subjects may have used a "sense of effort" (Merton, 1970) as a cue generated from the central nervous system. This would be most likely for the small breaths that did not involve significant movement of the chest wall.

Although the Weber fraction remained constant for both constrained and planned inspirations as a function of volume, it is still possible that different cues were utilized, depending on the size of the volume. This could be determined by altering the end expiratory position and then having subjects reproduce either the endpoint or the volume of the original breath.

\section{REFERENCE NOTE}

1. Mihevic, P., Gliner, J. A., \& Horvath, S. M. Perception of effort and respiratory sensitivity during exposure to ozone. Un. published manuscript, Institute of Environmental Stress, University of California, Santa Barbara, 1981.

\section{REFERENCES}

AXEN, K., \& HAAS, S. Effect of thoracic denervation on the ventilatory response to added loads in man. Federation Proceedings, 1979, 38, 1142

BAIRD, J., \& NomA, E. Fundamentals of scaling and psychophysics. New York: Wiley, 1978.

Bakers, J., \& Tenney, S. The perception of some sensations associated with breathing. Respiration Physiology, 1970, 10, $85-92$.

EDWARDS, A. Statistical methods for the behavioral sciences. New York: Holt, Rinehart \& Winston, 1964.

Matthews, P. Muscle afferents and kinaesthesia. British Medical Bulletin, 1977, 33, 137-142.

McCloskey, D. Kinesthetic sensibility. Physiological Reviews, 1978, 58, 763-820.

Merton, P. A. The sense of effort. In R. Porter (Ed.), Breathing: Hering-Breuer Centenary Symposium. London: Churchill, 1970.

Shannon, R. Phrenic nerve response to costovertebral joint movement. Federation Proceedings, 1979, 38, 1299.

Stelmach, G. E., Kelso, J. A. S., \& McCullagh, P. D. Preselection and response biasing in short-term motor memory. Memory \& Cognition, 1976, 4, 62-66.

Teghtsoonian, R. On the exponents in Stevens' law and the constant in Ekman's law. Psychological Review, 1971, 78, 71-80.

West, D., Ellis, C., \& CAmpbell, E. Ability of man to detect increases in his breathing. Journal of Applied Physiology, 1975, 39, 372-376.

Woodworth, R., \& Schloseng, H. Experimental psychology. New York: Holt, Rinehart \& Winston, 1965.

\section{NOTES}

1. The nature and purpose of the study and the risks involved were explained verbally and given on a written form to each subject prior to his or her voluntary consent to participate. The protocol and procedures for this study have been approved by the Committee on Activities Involving Human Subjects, of the University of California, Santa Barbara.

2. Woodworth and Schlosberg (1965) suggested that the standard deviation of the settings, if used consistently, would serve as an excellent measure of discrimination or in a test of Weber's law. However, we were also interested in comparing the Weber fraction from our data with those of other senses in order to determine the relative sensitivity of the respiratory system. Therefore, we utilized the standard deviation of the settings multiplied by .6745 , which yielded the probable deviation or probable error. In a normal distribution, the interval established by the mean, plus and minus one probable distribution, contains the middle $50 \%$ of the measures and is equivalent to the difference between the third quartile and the first quartile (Edwards, 1964). According to Woodworth and Schlosberg (1965), this corresponds to the difference limen from the method of limits.

(Received for publication June 27, 1980; revision accepted January 20,1981 .) 